\title{
Le bestiaire mélanésien. 100 représentationsde Didier Zanette
}

Isabelle Leblic

\section{OpenEdition}

1 Journals

\section{Édition électronique}

URL : http://journals.openedition.org/jso/6166

DOI : $10.4000 /$ jso.6166

ISSN : 1760-7256

\section{Éditeur}

Société des océanistes

\section{Édition imprimée}

Date de publication : 15 décembre 2010

Pagination : 269-270

ISBN : 978-2-85430-027-7

ISSN : 0300-953x

\section{Référence électronique}

Isabelle Leblic, "Le bestiaire mélanésien. 100 représentationsde Didier Zanette », Journal de la Société des Océanistes [En ligne], 130-131 | 2010, mis en ligne le 15 décembre 2010, consulté le 24 septembre 2020. URL : http://journals.openedition.org/jso/6166 ; DOI : https://doi.org/10.4000/jso.6166 
mineure, du titre : « étoffes cosmiques » qui tendrait à donner un sens exagérément astronomique à cette production, alors qu'il suffit de les considérer comme des « biens de prestige » dont l'usage se manifeste au cours des moments les plus solennels de la vie sociale. Il ne nous viendrait pas à l'idée de traiter les tissus de la vie monarchique ou impériale française comme des « textiles cosmiques », quels que soient les éléments de décoration qui y apparaissent. L'emphase du titre mise à part, le livre doit désormais être classé dans la catégorie des ouvrages de référence sur la question du tapa en Océanie.

Raymond MAYER, Université Omar Bongo de Libreville et Université de Lyon 2

ZanetTe Didier, 2010. Le bestiaire mélanésien. 100 représentations, Nouméa, DZ Productions, «Et si nous parlions l'Océanien ?», 160 p., bibliogr. indicative, une carte, table des 100 objets reproduits, lexique, nombreuses ill. couleur.

Sujet intéressant s'il en est mais mal illustré dans cet ouvrage où les photographies, ne sont pas toujours de qualité et montrent mal les objets, et dont le texte qui les accompagnent manquent de références à la bibliographie existante sur le sujet. La bibliographie, qui ne comporte que quinze références, est quasiment entièrement consacrée à " l'art » (huit des ouvrages mentionnés contiennent le mot art dans leur titre) ou aux collections muséales. Peu de références ethnographiques alors que le sujet ne peut être appréhendé à mon sens en faisant l'économie des apports de l'ethnologie, de l'ethnolinguistique et de l'importance des traditions orales en la matière. Puis, au fil de la lecture, on découvre en notes de bas de page quelques autres références qui seraient venues utilement compléter la bibliographie.

L'ouvrage est construit en sept chapitres faisant suite à l'introduction pp. 7-11): chapitre I «Aux débuts, les mythes... » (pp. 13-19); chapitre II « Dans l'océan » (pp. 21-45); chapitre III «Entre eaux saumâtres et terre » (pp. 47-65); chapitre IV « Sur terre, le roi cochon et les autres » (pp. 67-93); chapitre $\mathrm{V} « \mathrm{Au}$ pays des insectes» (pp. 95-101); chapitre VI «Le monde de la gent à plume » (pp. 103-131); chapitre VII «Et aujourd'hui?» (pp. 133-139), un index d'images (pp. 141-151) qui n'est en réalité qu'une table faite à l'aide de photos miniatures renvoyant à la page où l'objet est reproduit dans l'ouvrage et un glossaire (pp. 153-155). Pour finir, une page de références bibliographiques et un sommaire très peu indicatif qui est là en fait pour signaler les crédits photos, les artistes contemporains et les diverses mentions légales de l'ouvrage (un vrai fourre-tout).

Chaque chapitre commence par la reproduction d'une œuvre d'art contemporaine dont on trouve l'auteur dans le sommaire mais sans aucune précision ni de titre, ni de la nature de l'œuvre et encore moins sur l'artiste lui-même ! Dommage !

La page de préface pour introduire « l'importance de l'animal dans le bestiaire océanien » (p. 7) signée par Dominique Franchot (dont on ne nous dit pas qui il/elle est ${ }^{25}$ ) est bien limitée et nous place face à des lieux communs quand ce n'est pas des imprécisions pouvant conduire à de fausses interprétations, comme nous le verrons ci-dessous. L'introduction qui suit (pp. 7-11) ne nous satisfait guère plus. Ainsi, une telle phrase dans le premier paragraphe n'a guère de signification :

«En Nouvelle-Calédonie, on ne trouve plus, par exemple, qu'une espèce de mammifère la roussette et plus aucun reptile terrestre à l'exception toutefois du lézard. » (p. 7)

Ainsi, on ne sait pas à quelle période l'auteur fait référence avec ce « plus ». Si l'on se reporte au magnifique site internet endemia.nc, on trouve le serpent des Loyauté (Candoia bibroni) dit aussi boa du Pacifique et qui est un reptile terrestre (http://www.endemia. nc/faune/fiche1097.html - consulté le 3 avril 2010); côté lézard, leur variété endémique est immense. En $2003^{26}$, une recherche sur le site http://www.endemia. nc/ tel qu'il existait à cette date m'avait permis de recenser 67 espèces de lézards dont 59 endémiques, qui se répartissent en 42 espèces de Scincidae (dont 39 endémiques) et 25 espèces de Geckos (dont 20 endémiques appartenant à la famille Diplodactylidae qui comprend le genre Rhacodactylus cité par Leenhardt ; seules les cinq espèces de la famille Gekkonidae ne sont pas endémiques). Une telle phrase ne peut donc pas en rendre compte et laisser penser au lecteur non connaisseur que la faune calédonienne est d'une grande pauvreté, alors que quiconque s'intéresse à la Nouvelle-Calédonie en connaît la richesse endémique exceptionnelle.

En fait, chaque chapitre, après un paragraphe d'une banale généralité, ne fait que commenter les objets reproduits, sans forcément de liens entre les uns et les autres.

Beaucoup d'imprécisions, aucune information sur les objets collectés par l'auteur pour lesquels il aurait été utile de savoir où précisément il avait été trouvé, à quelle date et comment s'est faite l'acquisition et sur son usage particulier. Par exemple, l'objet 11, nommé «amulette» et dit «charme particulier» pour la pêche au dugong, nous est présenté sans autre précision sur les techniques de pêche de la région!

« Le lézard n'est pas toujours un ancêtre. Il est également l'objet de multiples légendes et de nombreux contes » (p. 65)

25. « Dominique Franchot était le directeur des Ressources humaines du Groupe ERAMET jusqu'en décembre 2009, c'est un passionné de voyages et d'art premier. Ses fonctions l'amenaient à venir régulièrement en Nouvelle-Calédonie pour la Société Le Nickel » (Isabelle Zanette, com. pers., 30/09/2010).

26. Voir la communication « Animaux, représentations totémiques et esprits des ancêtres dans quelques sociétés kanak de Nouvelle-Calédonie » que j'ai faite au colloque international le symbolisme des animaux - l'animal " clé de voûte " dans la tradition orale et les interactions homme-nature, organisé par le LACITO CNRS et l'IRD, à Villejuif (12-14/11/2003). 
Cette opposition rapide faite par l'auteur n'a pas toujours lieu d'être car, chez les Kanak par exemple, c'est aussi parce que le lézard représente l'autochtonie et les ancêtres des clans terriens qu'il est présent dans de nombreux contes et légendes. D'ailleurs, en commentant un bambou gravé du musée de NouvelleCalédonie, il écrit :

«Sans doute est-ce l'une de ces histoires qu'un artiste kanak a gravée sur un bambou, un lézard encerclé d'hommes qui cherchent à le capturer, à moins qu'ils ne dansent autour de lui » (p. 65)

Que voit-on sur ce morceau de bambou gravé ? Sans aucun doute une scène de la colonisation : au centre, à la verticale dans ce que l'on pourrait croire être une allée centrale, un lézard; des guerriers kanak d'un côté, qui semblent l'affronter ; de l'autre un personnage en costume représentant sûrement un Européen ; en bas une frise de fusils et en haut une case rectangulaire abritant deux hommes en face à face assis sur une chaise et entre eux, trois hommes habillés. De part et d'autre de la case, deux soldats armés montent la garde. Au-dessus, un médaillon avec un profil d'homme que Roger Boulay (sous presse) attribue à Napoléon III ${ }^{27}$. Ainsi pourrait-on analyser ce bambou gravé comme l'irruption de la colonisation s'opposant aux autochtones, en s'appuyant parfois sur les rivalités entre Kanak eux-mêmes. Ce passage sur le lézard est significatif de la méconnaissance de la société qui produit ces objets ! Il aurait pu citer le superbe catalogue de l'exposition Bambous kanak au MEG - et actuellement au musée de Nouvelle-Calédonie à Nouméa -, dont nous avons rendu compte dans le Jso 126-127 en Miscellanées Bambous kanak.

Pour finir, arrêtons-nous sur le dernier chapitre : « la rencontre avec la civilisation occidentale... » (p. 133) alors que 78 des objets présentés dans les pages précédentes sont du $\mathrm{XX}^{\mathrm{e}}$ siècle et $3 \mathrm{du} \mathrm{XXI}^{\mathrm{e}}$ siècle $^{28}$ ! Il en reste 19 pour le XIX ${ }^{\mathrm{e}}$ siècle $^{29}$ ! Cette rencontre se passa à des moments variables à partir en gros du milieu du XIX ${ }^{\mathrm{e}}$ siècle et peu des objets présentés ici sont donc vierges de tout contact, sans pour autant que leur présentation ne soit dénuée d'intérêt car les musées sont plein de ces objets produits au moment du contact. D'ailleurs l'auteur ne parle-t-il pas de «l'inculturation [sic !] des techniques et des procédés venus d'ailleurs » (p. 133), sans doute veut-il parler d'acculturation!

Une grosse déception donc à la lecture de ce beau livre (on regarde de belles images, c'est sûr !) qui manque de commentaires et de référence sur les objets.

\section{RÉFÉRENCES CITÉES}

Boulay Roger, sous presse. Le profil de « Napoléon III » \& Vue de profil, catalogue de l'exposition Bam- bous kanak au musée de Nouvelle-Calédonie, Nouméa, musée de Nouvelle-Calédonie, pp. 12-13 et p. 27.

Colombo Dougoud Roberta (éd.), 2008. Bambous kanak. Une passion de Marguerite LobsigerDellenbach, Genève, MEG, INFOLIO, Sources et témoignages 9, 184 p., bibliogr., glossaire, nombreuses illustrations (photographies en couleur ou noir et blanc, dessins au trait).

LeBLIC Isabelle, 2008. À propos de Bambous kanak. Une passion de Marguerite Lobsiger-Dellenbach, in Jean Trichet et Isabelle Leblic (éds), Journal de la Société des Océanistes 126-127 : Spécial environnement dans le Pacifique, pp. 311-317.

Isabelle LEBLIC, CNRS-LACITO, Villejuif

Musique du monde. Nouvelle-Calédonie. Voix des rivages et des montagnes, $\mathrm{CD}$ enregistré en Nouvelle-Calédonie et brochure de présentation français-anglais, 31 p., coll. Dominique Buscail, Buda musique.

Ce coffret musical est issu d'une collecte effectuée en février 2009 en Nouvelle-Calédonie par la productrice et le réalisateur de l'émission "Couleurs du monde " de France-Musique, Françoise Degeorges et Charles Le Gargasson, guidés par Emmanuel Tjibaou et Hervé Lecren, " en quête de l'esprit ancestral qui irrigue la société kanak contemporaine » (p. 3) avec l'aide du Centre Jean-Marie Tjibaou, du Conservatoire de musique de Nouvelle-Calédonie, du Pôle d'exports des musique et des arts de Nouvelle-Calédonie et de la Maison de la Nouvelle-Calédonie à Paris. Enregistrés in vivo (avec les chants d'oiseaux en arrière-plan avant les voix), du nord au sud de la Grande Terre, les vingt-deux morceaux rassemblés ici présentent un éventail de chants en langues kanak. À l'écoute, on se trouve transporté à $20000 \mathrm{~km}$ de Pars, en terre kanak, au milieu de ces femmes et hommes qui chantent les $a e ́-a e ́$ célébrant l'emblème totémique lézard, des événements tels qu'une inondation dans la tribu de Bwara à Canala ou que les vols de terres par les colons, des chants polyphoniques dans lesquels les Kanak excellent, des comptines chantées pour les enfants ou encore des tempérances rendant hommage aux militants tombés pour la lutte indépendantiste. Moi qui ai participé à maintes festivités des écoles populaires kanak de la région de Canala, j'ai écouté à l'époque de nombreux chants consacrés à garder en mémoire les événements dramatiques de l'époque. Un tel disque ne pouvait se faire sans le traditionnel discours sur le bois nommé de façon impropre « généalogie » en français.

27. «Sur cette pièce [MNC 98.5.1], les images sont strictement organisées autour de la figure de Napoléon III gravée à son sommet. Le dessin s'inspire sans nul doute du profil de l'Empereur lisible sur les monnaies de l'époque. » (Boulay, sous presse : 12).

28. À ce titre, d'aucuns notent que ces objets les plus modernes et pouvant nous paraître hideux restent instructifs dans leur genre.

29. La petite dizaine d'objets anciens qui est présentée ici mériteraient une enquête. 